\title{
ASPECTS OF IDENTIFICATION OF ELASTIC-PLASTIC PARAMETERS USING HEURISTIC ALGORITHMS
}

\author{
M. Vaz $\mathrm{Jr}^{1}$, E. L. Cardoso ${ }^{2}$ \\ ${ }^{1}$ Department of Mechanical Engineering, State University of Santa Catarina \\ (M.Vaz@joinville.udesc.br) \\ ${ }^{2}$ Department of Mechanical Engineering, State University of Santa Catarina
}

\begin{abstract}
The intense research in the development of new constitutive models has faced the challenge of devising strategies to determine the corresponding material parameters. The literature has shown a steady growth in application of parameter identification based on optimization techniques to a wide range of engineering problems. Within this framework, in recent years, parameter identification schemes using heuristic approaches have been proposed as possible alternatives to classical identification procedures mainly due to their potential ability to avoid local minima, insensitivity to the order of magnitude of parameters and easy parallelisation. The present work shows that Particle Swarm Optimization, as an example of such methods, can also be successfully applied to identification of inelastic parameters and presents remarkable characteristics when compared to Genetic Algorithms.
\end{abstract}

Keywords: Parameter Identification, Particle Swarm Optimization, Genetic Algorithm.

\section{INTRODUCTION}

The ongoing development of commercial packages contemplating numerical modelling of elastic-plastic problems at finite strains has made possible the simulation of a wide range of metal forming processes, such as forging, extrusion and deep drawing amongst others. Notwithstanding, the success of a simulation is strongly associated with material modelling and corresponding parameters. Such pressing need has fostered the development of new parameter identification strategies based upon optimization strategies. These identification techniques are mostly gradient-based optimization procedures and heuristic approaches. The former requires computation of the gradient of the objective function and has been indicated to convex problems. However, parameter identification of complex constitutive relations generally renders non-convex problems causing gradient-based optimization schemes to fail at finding the global minimum. Heuristic methods, nevertheless, are able to avoid local minima in non-convex optimization and allow easy parallelisation, mitigating their higher computational cost. 
The literature shows many works addressing application of gradient-based optimization procedures to identification of elastic-plastic material parameters (see Muñoz-Rojas et al. [1] and references therein). It is noteworthy that most authors report some convergence difficulties (to the global minimum) when using damage, anisotropic or other complex constitutive relation, giving rise to hybrid strategies or adopting purely heuristic techniques. For instance, Artificial Neural Networks (ANN) was used by Abendroth and Kuna [2] to identification of a Gurson-type damage model. Aguir et al. [3], aiming at anisotropic materials, utilised also ANN as an alternative to the Finite Element calculations to evaluate the objective function within the Genetic Algorithm (GA). A combination of Genetic Algorithms and gradientbased optimization methods was also proposed to solving parameter identification problems [4,5]. In a first stage, Genetic Algorithms are applied in order to reduce the search space to a region sufficiently close to the minimum. The gradient-based technique is applied next using the best individual of the last generation as the initial parameter estimate. The relatively slow convergence rate has discouraged sole use of GA to determine the final parameters. In recent years, Particle Swarm Optimization (PSO) has been proposed to general optimization problems [6,7] as an alternative to evolutionary algorithms owing to its high success rate and stability. This method is based on Swarm Intelligence and attempts to mimic the social behaviour of populations, such as bird flocking and fish schooling. Following the route on using gradient-free optimization techniques to identification of inelastic parameters, this work, based upon previous investigations [8], presents an assessment of the PSO method and a brief comparative study between GA and PSO techniques within the framework of a classical von Mises material. Apparently simple, the test case makes possible to establish the viability of using PSO in this class of problems, providing robustness and accuracy insights.

\section{THE OPTIMIZATION PROBLEM}

In elastoplasticity, parameter identification consists of finding material parameters (elastic and/or inelastic) of the constitutive model using techniques of so-called inverse problems. The approach used in the present work is based on unconstrained optimization, which is generally defined as

$$
\begin{array}{lll}
\text { Minimize } & g_{0}(\boldsymbol{p}) & \boldsymbol{p} \in R^{n} \\
& p_{i}^{\text {inf }} \leq p_{i} \leq p_{i}^{\text {sup }} & i=1 \ldots n
\end{array},
$$

in which $\boldsymbol{p}=\left[\begin{array}{llllll}p_{1} & p_{2} & \ldots & p_{i} & \ldots & p_{n}\end{array}\right]^{T}$ is the design vector containing $n$ design variables (each design variable, $p_{i}$, corresponds to a given material parameter), and $p_{i}^{\text {sup }}$ and $p_{i}{ }^{\text {inf }}$ are the upper and lower bounds of the design variables, respectively. The objective function (or fitness), $g_{0}(\boldsymbol{p})$, to be minimized represents an error measure between the experimental and corresponding computed response (the latter is obtained using Finite Elements [9]). This work uses the relative quadratic difference between the experimental measure, $R^{E x p}$, and numerical response, $R^{\text {FEM. }}(\boldsymbol{p})$ 


$$
g_{0}(\boldsymbol{p})=\sqrt{\frac{1}{N} \sum_{q=1}^{N}\left(\frac{R_{q}^{E x p}-R_{q}^{F E M}}{R_{q}^{E x p}}\right)^{2}} .
$$

where $N$ is the number of experimental points.

Parameter identification may yield a non-convex and highly nonlinear optimization problem thereby hindering determination of a global minimum, especially when using gradient-based optimization strategies. In recent years the literature shows increasing application of soft computing algorithms to this class of problems owing to their potential capacity to avoid local minima and to circumvent possible lack of convergence of the elastic-plastic problem itself [8]. Neural Networks algorithms (e.g. Artificial Neural Networks), Evolutionary (e.g. Genetic Algorithms), Swarm Intelligence (e.g. Particle Swarm Optimization and Ant Colony Optimization) are the most referred soft computing strategies for general engineering problems. However, there are relatively few attempts to using such methods to identification of elastic-plastic material parameters.

\subsection{Particle Swarm Optimization}

Particle Swarm Optimization was introduced by Eberhart and Kennedy [6,7] using concepts of social behaviour of populations. The technique proved to be successful in many engineering problems, such as design and optimization of communication / electricity networks, economic load dispatching and electric motors; robotics, supply chain management, job and resource allocation, and system identification amongst many other applications [1012]. In spite of such wide usage spectrum, application of PSO techniques to identification of material parameters is relatively new (see, for instance, references [13-15] for identification of thermal parameters).

In PSO techniques, the population comprises particles to which are applied velocity operators in an attempt to simulate a combination of individual cognitive abilities and social interactions. In a first step, the initial population is randomly defined within the parameter lower and upper limits. The scheme attributes velocities to each particle taking into account (i) its inertia, (ii) personal history and (iii) neighbourhood effect.

(i) Inertia: represents the tendency of a particle to follow its previous direction;

(ii) Personal history: the location in the search space which results its best fitness - the cognitive effect;

(iii) Neighbourhood effect: effect of the best fitness of neighbouring individuals - the social effect.

It is relevant to mention that several different variants of the method have been proposed, most of which defining new rules of particle interactions and neighbourhood conditions [16]. This work addresses the classical implementation of the PSO technique, described as follows:

Initially, a random population and corresponding initial velocities are generated

$$
\boldsymbol{p}^{(0)}=\left\{\boldsymbol{p}_{1}^{(0)} \boldsymbol{p}_{2}^{(0)} \ldots \boldsymbol{p}_{m}^{(0)} \ldots \boldsymbol{p}_{n_{p}}^{(0)}\right\} \quad \text { and } \quad \boldsymbol{v}^{(0)}=\left\{\boldsymbol{v}_{1}^{(0)} \boldsymbol{v}_{2}^{(0)} \ldots \boldsymbol{v}_{m}^{(0)} \ldots \boldsymbol{v}_{n_{p}}^{(0)}\right\}
$$


in which $n_{p}$ is the population size and

$$
\boldsymbol{p}_{m}^{(0)}=\left[\begin{array}{lllllll}
p_{1}^{(0)} & p_{2}^{(0)} & \ldots & p_{i}^{(0)} & \ldots & p_{n}^{(0)}
\end{array}\right]^{T} \text { and } \boldsymbol{v}_{m}^{(0)}=\left[\begin{array}{llllll}
v_{1}^{(0)} & v_{2}^{(0)} & \ldots & v_{i}^{(0)} & \ldots & v_{n}^{(0)}
\end{array}\right]^{T}
$$

where $p_{i}$ is the particle coordinate in the search space (a material parameter), $v_{i}$ is the corresponding velocity component, and $n$ is the total number of material parameters. The method establishes subsequent computation of new velocities and locations according to pre-defined rules and operators. The most widely used PSO operators define the new particle velocity, $\boldsymbol{v}^{(k+1)}$, and location, $\boldsymbol{p}^{(k+1)}$, as

$$
\begin{aligned}
& \boldsymbol{v}^{(k+1)}=\overbrace{w \boldsymbol{v}^{(k)}}^{\text {Inertia }}+\overbrace{\boldsymbol{U}\left(0, \varphi_{1}\right) \otimes\left(\boldsymbol{p}_{i b}^{(k)}-\boldsymbol{p}^{(k)}\right)}^{\text {Cognitive }}+\overbrace{\boldsymbol{U}\left(0, \varphi_{2}\right) \otimes\left(\boldsymbol{p}_{g b}^{(k)}-\boldsymbol{p}^{(k)}\right)}^{\text {Social }}, \\
& \boldsymbol{p}^{(k+1)}=\boldsymbol{p}^{(k)}+\boldsymbol{v}^{(k+1)}
\end{aligned}
$$

where the superscript $(k)$ indicates time step, $\boldsymbol{p}_{i b}$ and $\boldsymbol{p}_{g b}$ are the individual and global best locations, respectively, $w$ is the inertia parameter, and $\boldsymbol{U}\left(0, \varphi_{1}\right)$ and $\boldsymbol{U}\left(0, \varphi_{2}\right)$ represent vectors of random numbers defined in the interval $\left[0, \varphi_{1}\right]$ and $\left[0, \varphi_{2}\right]$, where $\varphi_{1}$ and $\varphi_{2}$ are the cognitive and social parameters, respectively. The operation $\langle\cdot\rangle \otimes\langle\cdot\rangle$ indicates a component-wise multiplication.

In addition to Equations (5), velocity and boundary control are required in order to avoid excessive particle dispersion and boundary violation. In the present work, velocity components are restricted to a fraction of $\left(p_{i}^{\text {sup }}-p_{i}^{\text {inf }}\right)$ for each parameter, so that

$$
\text { If }\left|v_{i}^{(k+1)}\right|>v_{i}^{\max } \Rightarrow\left|v_{i}^{(k+1)}\right| \leftarrow v_{i}^{\max }, \quad \text { where } \quad v_{i}^{\max }=w_{i}\left(p_{i}^{\text {sup }}-p_{i}^{\text {inf }}\right)
$$

is the maximum velocity component, where $\left|\boldsymbol{v}_{i}^{(k+1)}\right|$ is the absolute value of the velocity component, and $w_{i} \in[0,0.5]$ is the velocity restriction parameter. Noticeably, there are other velocity control schemes, amongst which Clerc and Kennedy's [17] constriction parameter is the most referred. However, this strategy was not sufficient to waive using boundary control in the present identification problem.

The random character of the velocity operators allied to an eventual proximity of a particle to the search space boundary may cause an individual to violate the pre-defined lower or upper bounds, $p_{i}^{\text {sup }}$ and $p_{i}{ }^{\text {inf }}$. The present scheme enforces a boundary control borrowed from GA, which imposes component-wise limits, i.e., an individual component, $p_{i}$, is reset at the boundary,

$$
\begin{aligned}
& \text { If } p_{i}^{(k+1)}>p_{i}^{\text {sup }} \quad \Rightarrow \quad p_{i}^{(k+1)} \leftarrow p_{i}^{\text {sup }} \\
& \text { If } p_{i}^{(k+1)}<p_{i}^{\text {inf }} \quad \Rightarrow \quad p_{i}^{(k+1)} \leftarrow p_{i}^{\text {inf }}
\end{aligned} .
$$

\subsection{Convergence and accuracy measures}

Convergence and accuracy measures in heuristic algorithms have prompted a healthy discussion in recent years. Gradient-based optimization schemes can naturally use the norm of 
the gradient of the objective function to evaluate convergence. However, GA and PSO methods do not demand computation of the gradient and, therefore, require alternative convergence assessment (or simply stopping criteria). In general, the (a) total number of generations, $k^{\max },(b)$ the difference between the best and worst individuals or else $(c)$ the difference between the best fitness of consecutive generations have been largely used in conjunction with Genetic Algorithms. Similarly, a normalised fitness convergence index, $\phi_{g}$, computed using the worst and best particles can also be defined as

$$
\phi_{g}^{(k)}=\frac{g_{0}\left(\boldsymbol{p}^{(k)}\right)^{\text {worst }}-g_{0}\left(\boldsymbol{p}^{(k)}\right)^{\text {best }}}{g_{0}\left(\boldsymbol{p}^{(0)}\right)^{\text {worst }}-g_{0}\left(\boldsymbol{p}^{(0)}\right)^{\text {best }}} .
$$

The characteristics of PSO algorithms make possible to define stopping / convergence criteria based upon dispersion of particles or corresponding velocities. As the optimization process evolves, particles tend to cluster together, reducing velocity and dispersion, so that

$$
\phi_{d}^{(k)}=\frac{\sum_{m=1}^{n_{s}}\left|\boldsymbol{p}_{s b}^{(k)}-\boldsymbol{p}_{m}^{(k)}\right|}{\sum_{m=1}^{n_{s}}\left|\boldsymbol{p}_{s b}^{(0)}-\boldsymbol{p}_{m}^{(0)}\right|},
$$

where $|\langle\cdot\rangle|$ indicates the Euclidean norm and $\boldsymbol{p}_{s b}{ }^{(k)}$ is the best particle of time step $(k)$. The dispersion index, $\phi_{d}$, represents the relative mean distance between particles and the best individual of the step $k$. Convergence in GA and PSO schemes can also be measured based on a fraction of the total population, $n_{s}$, defined in order to exclude non-physical individuals from computation of convergence indices (although defined within predefined limits, some combination of parameters may render unrealistic individuals or particles, hampering convergence of the direct elastic-plastic problem, especially in early optimization stages).

An alternative convergence indicator can be expressed in terms of the mean equivalent number of bits, $\bar{n}_{b}^{e}$, of the algorithm, which measures the average resolution in each time step or generation, $k$. Although the present PSO implementation does not use a binary approach, $\bar{n}_{b}^{e}$ has proved to be a good quality indicator of the final parameters. In GA algorithms, the relation of the resolution, $\Delta p_{i}$, upper and lower limits of a parameter, $p_{i}^{\text {sup }}$ and $p_{i}^{\text {inf }}$, and number of bits, $n_{b}$, is well established [18]. Similarly, a mean equivalent number of bits can be computed in each step/generation using the average resolution of parameters, $\Delta \bar{p}_{i}^{e}$, as

$$
\bar{n}_{b}^{e^{(k)}}=\frac{1}{n} \sum_{i=1}^{n} n_{i}^{e^{(k)}}, \text { where } n_{i}^{e^{(k)}}=\frac{\ln \left(\frac{p_{i}^{\text {sup }}-p_{i}^{\mathrm{inf}}}{\Delta \bar{p}_{i}^{e^{(k)}}}+1\right)}{\ln (2)} \text { and } \Delta \bar{p}_{i}^{e^{(k)}}=\frac{p_{i}^{\max (k)}-p_{i}^{\min (k)}}{n_{s}-1}
$$

in which $n_{i}^{e}$ is the equivalent number of bits of a parameter, and $p_{i}^{\max }$ and $p_{i}^{\text {min }}$ are, respectively, the maximum and minimum values of each parameter in a given generation $k$. As the optimization evolves, GA individuals group closer to the minimum, progressively narrowing $p_{i}{ }^{\max }$ and $p_{i}{ }^{\min }$. The mean equivalent number of bits accounts for such narrowing effect and 
represents the effective resolution of the algorithm at certain stage of the identification procedure. Early in the process, the population presents large dispersion, reflected also in large values of $\Delta \bar{p}_{i}^{e}$ and small equivalent number of bits. In later generations, $\bar{n}_{b}^{e}$ approaches the theoretical resolution, i.e. $\bar{n}_{b}^{e} \rightarrow n_{b}$. Therefore, such assessment makes possible to objectively determine how close the algorithm is able to achieve the theoretical number of bits, i.e. it gives an alternative indication of the rate of convergence and quality of parameters. In addition, the mean equivalent number of bits can also be used to compare GA/PSO techniques.

\section{NUMERICAL EXAMPLES}

The present work addresses the classical von Mises material in an attempt to gain further insights on this class of identification problems and to establish eventual advantages or disadvantages of the PSO algorithm when compared to GA. This work emphasises some aspects of convergence assessment and presents comparative results against GA using the tensile test data presented by Ponthot and Kleinermann [19], corresponding to a special steel used in piping manufacture for the nuclear industry (Steel A-533, Grade B, Class 1). The gauge length and initial radius of the specimen are $r=6.413 \mathrm{~mm}$ and $2 \times \ell_{0}=53.342 \mathrm{~mm}$. The Finite Element mesh assumes revolution symmetry and contains 400 elements and 451 nodes with refinement at the necking region (similar to reference [19]). Modified Voce's [20] yield stress curve is also used in the present simulations,

$$
\sigma_{Y}=\sigma_{0}+\zeta \varepsilon_{p}+\left(\sigma_{\infty}-\sigma_{0}\right)\left[1-\exp \left(-\delta \varepsilon_{p}\right)\right]
$$

where $\sigma_{\infty}, \sigma_{0}, \zeta$ and $\delta$ are the parameters to be determined. The Young modulus and Poisson's ratio are assumed $E=206.9 G P a$ and $v=0.29$, respectively.

Table 1. GA / PSO lower and upper limits, and BFGS initial estimate and final parameters.

\begin{tabular}{ccccccc} 
& \multicolumn{5}{c}{ Parameters } \\
\cline { 2 - 5 } & $\sigma_{0}[\mathrm{MPa}]$ & $\sigma_{\infty}[\mathrm{MPa}]$ & $\zeta[\mathrm{MPa}]$ & $\delta$ & $g_{0}(\boldsymbol{p})$ \\
\hline \multirow{2}{*}{ Lower limit, $p_{i}^{\text {inf }}$} & 200 & 300 & 100 & 10 & $0.53981608642 \times 10^{0}$ \\
\multicolumn{2}{l}{ Upper limit, $p_{i}^{\text {sup }}$} & 800 & 1000 & 700 & 50 & $0.55331814228 \times 10^{0}$ \\
\hline \multirow{2}{*}{ BFGS } & Initial & 500 & 700 & 400 & 30 & $0.88655939242 \times 10^{-1}$ \\
& Final & 471.2533 & 678.1928 & 218.1305 & 15.52408 & $0.88181306615 \times 10^{-2}$ \\
\hline
\end{tabular}

The present test case was also solved using the BFGS gradient-based method [21] in order to obtain a reference solution. This strategy provides verification grounds for the PSO and GA methods, making possible to determine the final fitness and inelastic parameters with verifiable accuracy $\left(\boldsymbol{\phi}_{B F G S}^{\text {conv }}=\left|\nabla g_{0}\left(\boldsymbol{p}^{(k)}\right)\right| /\left|\nabla g_{0}\left(\boldsymbol{p}^{(0)}\right)\right|<1 \times 10^{-5}\right)$. It is worthy to highlight that the BFGS method was able to solve successfully the proposed inverse problem provided the initial parameter estimates fall within a given envelope. This behaviour is typical of gradientbased optimization procedures which require initial estimates sufficiently close to the final parameters. Table 1 presents the PSO / GA upper and lower limits and the initial estimates for 
the BFGS scheme - noticeably, the BFGS method fails to converge when using the upper / lower limits as initial estimates. Figure 1 illustrates the load - elongation curve computed using the material parameters presented in Table 1.

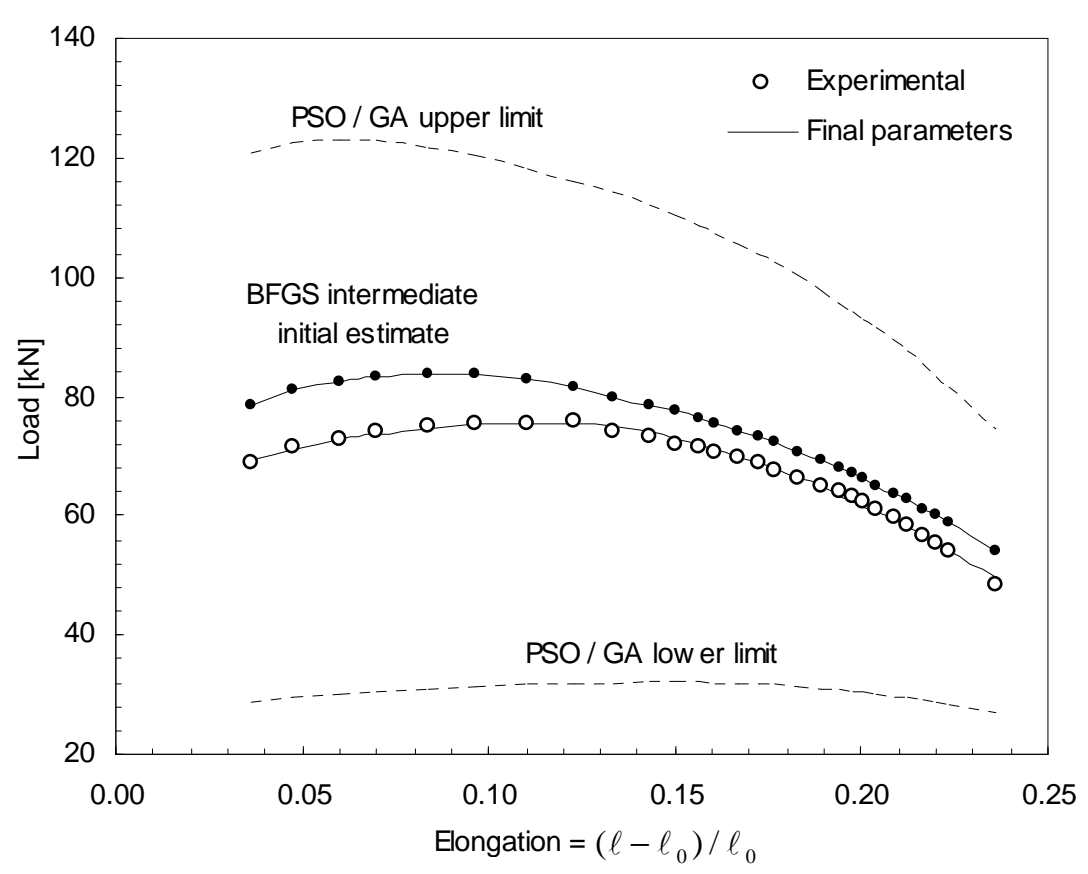

Figure 1. Experimental and numerical load curves for the tensile test.

The PSO technique requires definition of the inertia, $w$, cognitive, $\varphi_{1}$, and social parameters, $\varphi_{2}$. In a previous study, the authors investigated the influence of the PSO control parameters in the identification process, concluding that excessively smaller values lead to sub-optimal solutions and larger values cause swarm instability and convergence failure [8]. The recommended control parameters were $w \in[0.4,0.8]$ and $\varphi_{1}=\varphi_{2} \in[0.7,1.8]$. In the present study the weight parameters are assumed $w=0.5$ and $\varphi_{1}=\varphi_{2}=1.0$. Moreover, convergence of the PSO method is assumed for $n_{s}=80 \%$ when the fitness convergence and particle dispersion indices reach $\phi_{g}^{\text {conv }}=10^{-10}$ and $\phi_{d}^{\text {conv }}=10^{-6}$, respectively .

\subsection{Influence of parameters on the identification process}

The success of an identification process is reflected by determination of accurate parameters combined with smaller number of fitness computation. Although no sensitivity analysis is required by PSO schemes, a single or combination of material parameters can substantially affect the identification process. This example illustrates this issue by determining two out of a 4 parameter set (the parameters obtained using the BFGS method are used to complete the set), as indicated in Table 2, using a population size $n_{p}=10$. The simulations show that all cases, except Case (3), present very similar convergence behaviour, with accuracy for parameters and fitness in the $7^{\text {th }}$ and $10^{\text {th }}$ significant digits, respectively, when compared against the BFGS solution. 
Table 2. Influence of parameters: population $n_{p}=10$.

Parameter

\begin{tabular}{|c|c|c|c|c|c|}
\hline Case & $\sigma_{0}[\mathrm{MPa}]$ & $\sigma_{\infty}[\mathrm{MPa}]$ & $\zeta[\mathrm{MPa}]$ & $\delta$ & $g_{0}(\boldsymbol{p})$ \\
\hline$B F G S$ & 471.2533 & 678.1928 & 218.1305 & 15.52408 & $0.88181306615 \times 10^{-2}$ \\
\hline Case (1) & 471.2533 & $678.192 \underline{7}$ & $B F G S$ & $B F G S$ & $0.881813066 \underline{\mathbf{5 4}} \times 10^{-2}$ \\
\hline Case (2) & 471.2533 & $B F G S$ & 218.1305 & $B F G S$ & $0.881813066 \underline{\underline{16}} \times 10^{-2}$ \\
\hline Case (3) & $471.25 \underline{21}$ & $B F G S$ & $B F G S$ & $15.524 \underline{18}$ & $0.881813066 \underline{\underline{24}} \times 10^{-2}$ \\
\hline Case (4) & $B F G S$ & $678.192 \underline{7}$ & $B F G S$ & $15.5240 \underline{7}$ & $0.881813066 \underline{\underline{29}} \times 10^{-2}$ \\
\hline Case (5) & $B F G S$ & $B F G S$ & $218.130 \underline{6}$ & $15.5240 \underline{9}$ & $0.881813066 \underline{64} \times 10^{-2}$ \\
\hline Case (6) & $B F G S$ & $678.192 \underline{9}$ & $218.130 \underline{\mathbf{3}}$ & $B F G S$ & $0.881813066 \underline{\underline{22}} \times 10^{-2}$ \\
\hline
\end{tabular}

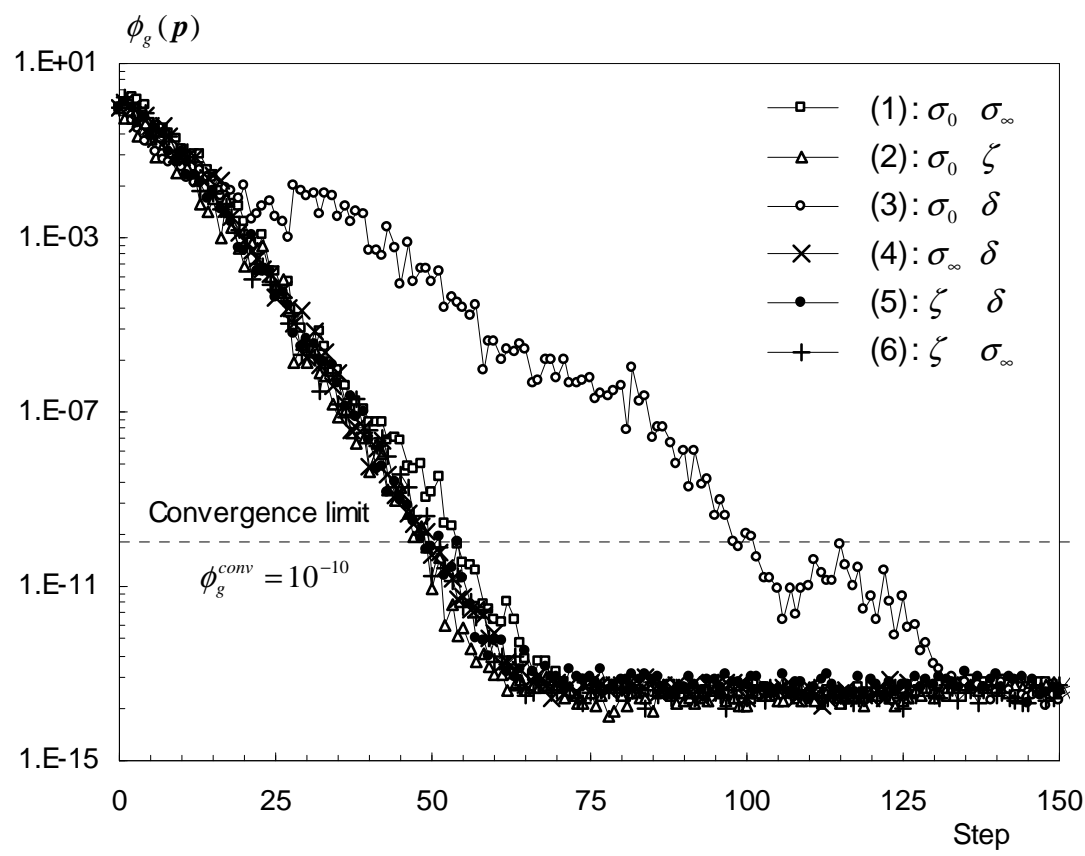

Figure 2. Convergence process evaluated using the normalised fitness index, $\phi_{g}$.

Figures 2 and 3 present the convergence process based on the normalised fitness and dispersion indices. The results show that the combined effect of parameters $\sigma_{\mathrm{o}}$ and $\delta$ - Case (3) - yields higher influence on the identification evolution, requiring larger population sizes to guarantee higher accuracy (to match the other cases, as illustrated in Table 2). Such behaviour indicates that, in this case, the population size $n_{p}=10$ represents the accuracy threshold, under which one would obtain convergence to sub-optimal parameters. It is also interesting to note that the higher influence of the parameters $\sigma_{\mathrm{o}}$ and $\delta$ are not due to the difference in the order of magnitude of parameters $\left(\sigma_{\mathrm{o}} \sim 10^{8}\right.$ and $\left.\delta \sim 10^{1}\right)$, since the combination $\left[\begin{array}{ll}\sigma_{\infty} & \delta\end{array}\right]^{T}$ and $[\zeta \quad \delta]^{T}$ presents similar magnitude differences but the expected convergence behaviour. Therefore, the higher influence of the combination $\left[\begin{array}{lll}\sigma_{\mathrm{o}} & \delta\end{array}\right]^{T}$ can be credited to effect of such parameters on the physics of the plastic deformation process. 


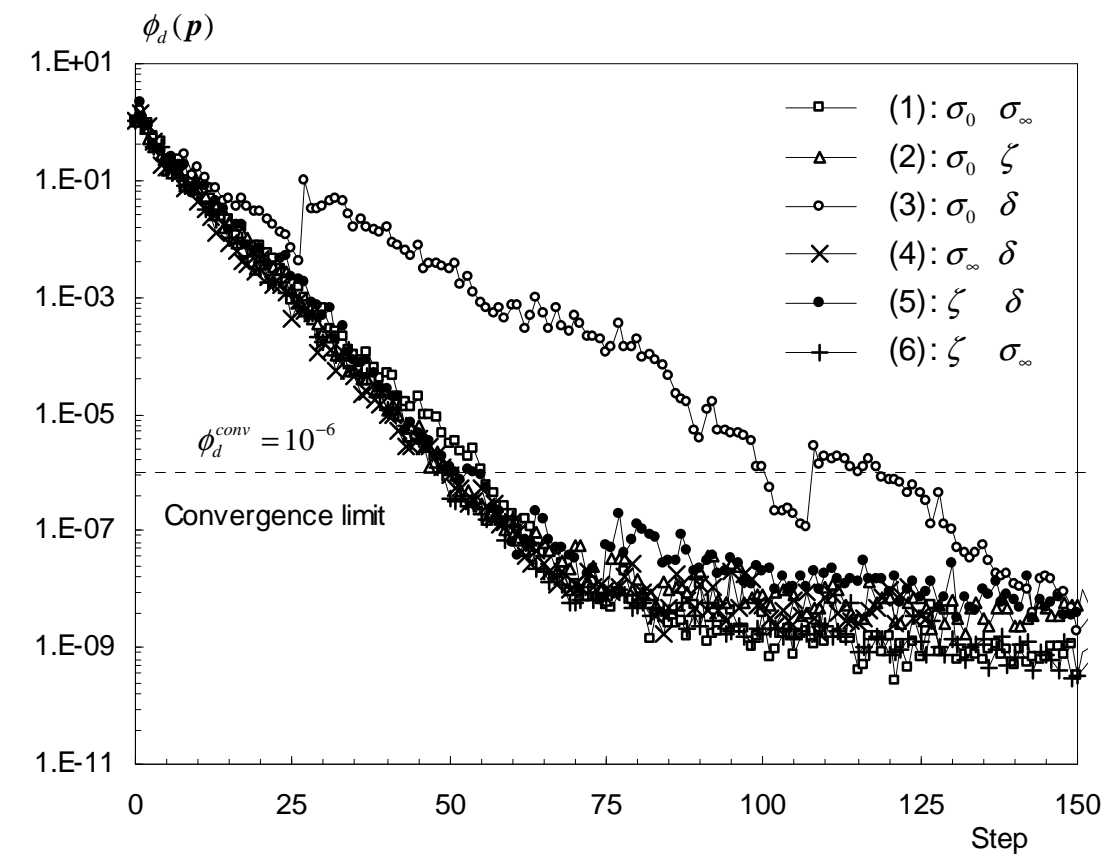

Figure 3. Convergence process evaluated using the dispersion index, $\phi_{d}$.

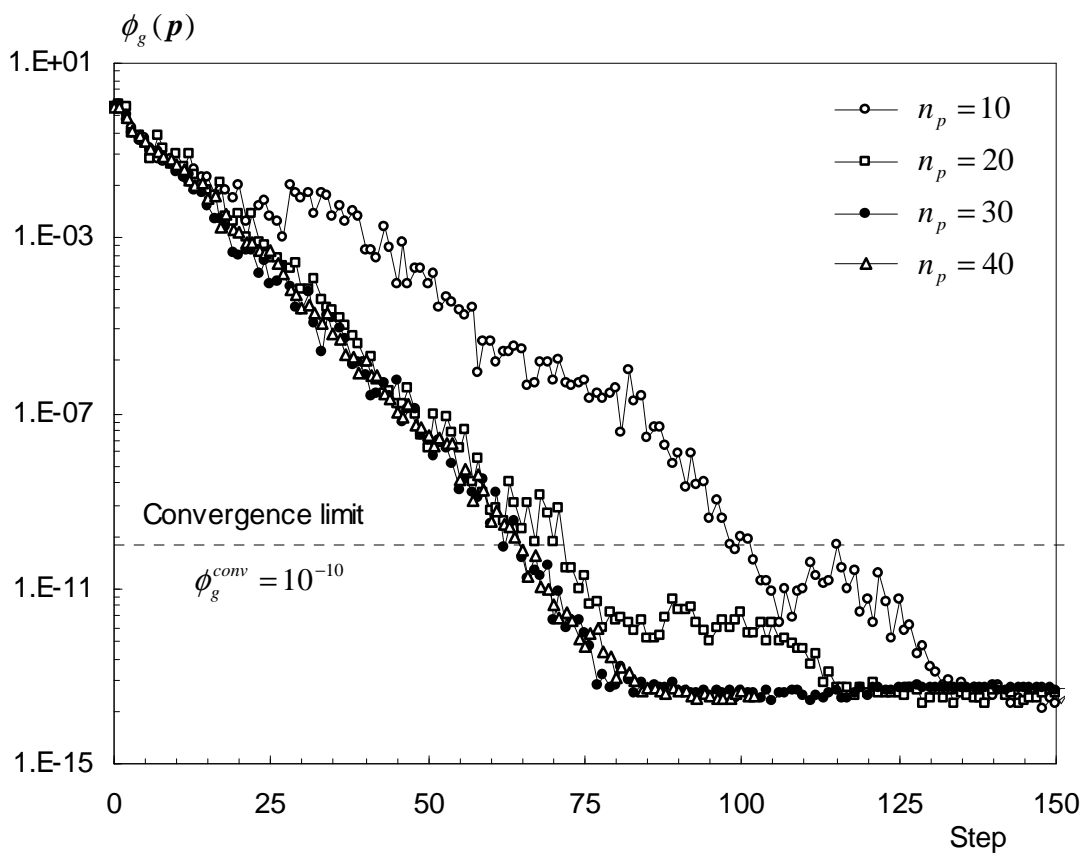

Figure 4. Effect of the population size to obtaining $\left[\sigma_{\mathrm{o}} \delta\right]^{T}$.

The aforementioned influence of the population size is illustrated in Figure 4 and Table 3, which show, respectively, the convergence process and final parameters for Case (3). As the population size increases, evolution of the optimization process approximates the expected behaviour (see Figure 4). However, it is important to emphasise that in all cases convergence was achieved with acceptable accuracy. 
Table 3. Influence of the population size to identification of $\left[\sigma_{\mathrm{o}} \delta\right]^{T}$.

\begin{tabular}{cccccc}
\cline { 2 - 5 } Population & $\sigma_{0}[\mathrm{MPa}]$ & $\sigma_{\infty}[\mathrm{MPa}]$ & $\zeta[\mathrm{MPa}]$ & $\delta$ & $g_{0}(\boldsymbol{p})$ \\
\hline$B F G S$ & 471.2533 & 678.1928 & 218.1305 & 15.52408 & $0.88181306615 \times 10^{-2}$ \\
\hline$n_{p}=10$ & $471.25 \underline{\mathbf{1}}$ & $B F G S$ & $B F G S$ & $15.524 \underline{\mathbf{1 8}}$ & $0.881813066 \underline{\mathbf{2 4}} \times 10^{-2}$ \\
$n_{p}=20$ & $471.25 \underline{\mathbf{4 6}}$ & $B F G S$ & $B F G S$ & $15.523 \underline{\mathbf{9 8}}$ & $0.881813066 \underline{\mathbf{4 2}} \times 10^{-2}$ \\
$n_{p}=30$ & $471.253 \underline{\mathbf{0}}$ & $B F G S$ & $B F G S$ & $15.524 \underline{\mathbf{1 0}}$ & $0.8818130661 \underline{\mathbf{4}} \times 10^{-2}$ \\
$n_{p}=40$ & $471.253 \underline{\mathbf{1}}$ & $B F G S$ & $B F G S$ & $15.524 \mathbf{1 1}$ & $0.8818130661 \underline{\mathbf{4}} \times 10^{-2}$ \\
\hline
\end{tabular}

\subsection{Influence of number of parameters}

The previous results indicate that the nature of a single material parameter affects the convergence process, thereby suggesting an ensuing significant influence of number of parameters upon the optimization procedure. Therefore, this example presents the convergence evolution of the PSO method when identifying parameters $(i)\left[\sigma_{\mathrm{o}}\right]$, (ii) $\left[\begin{array}{ll}\sigma_{\mathrm{o}} & \delta\end{array}\right]^{T}$, (iii) $\left[\begin{array}{lll}\sigma_{\mathrm{o}} & \zeta & \delta\end{array}\right]^{T}$ and $(i v)\left[\begin{array}{llll}\sigma_{\mathrm{o}} & \sigma_{\infty} & \zeta & \delta\end{array}\right]^{T}$. In cases (ii)-(iii), parameters $\sigma_{\mathrm{o}}$ and $\delta$ were selected owing to their higher influence on the optimization procedure. The remaining parameters in cases (i)-(iii) are those computed by the BFGS method.

The behavioural dynamics of particle interactions becomes evident in this example. As the dimension of the optimization hyperspace increases, the tendency of particles to converge towards a sub-optimal solution also increases. This effect can be counter-balanced by larger population sizes, which cause the global exploration capacity of the algorithm to grow. The obvious drawback is the increase of the computational cost (larger number of fitness evaluations), as highlighted in Figure 5. It is interesting to note that, when determining all 4 parameters, 160 particles is able to avoid sub-optimal solutions, achieving convergence after almost 30,000 fitness computation.

Table 4. Final converged parameters for Cases $(i)-(i v)$.

\section{Parameter}

\begin{tabular}{ccccccc}
\hline Case & & $\sigma_{0}[\mathrm{MPa}]$ & $\sigma_{\infty}[\mathrm{MPa}]$ & $\zeta[\mathrm{MPa}]$ & $\delta$ & $g_{0}(\boldsymbol{p})$ \\
\hline BFGS & & 471.2533 & 678.1928 & 218.1305 & 15.52408 & $0.88181306615 \times 10^{-2}$ \\
\hline (i) 1 Parameter & $n_{p}=5$ & 471.2533 & $B F G S$ & $B F G S$ & $B F G S$ & $0.88181306615 \times 10^{-2}$ \\
(ii) 2 Parameters & $n_{p}=20$ & $471.25 \underline{\mathbf{4 6}}$ & $B F G S$ & $B F G S$ & $15.523 \underline{\mathbf{9 8}}$ & $0.881813066 \underline{\mathbf{4 2}} \times 10^{-2}$ \\
(iii) 3 Parameters & $n_{p}=40$ & 471.2534 & $B F G S$ & 218.1305 & $15.5240 \underline{\mathbf{7}}$ & $0.88181306615 \times 10^{-2}$ \\
(iv) 4 Parameters & $n_{p}=160$ & $471.2 \underline{\mathbf{3 5 5}}$ & 678.1868 & 218.1488 & $15.52 \underline{\mathbf{6 3 0}}$ & $0.8818130 \underline{\mathbf{4 1 8 6}} \times 10^{-2}$ \\
\hline
\end{tabular}

The population sizes, $n_{p}$, indicated in Table 4 for cases (i)-(iii) guarantee convergence to the optimal solutions with very good accuracy (differences in the $6^{\text {th }}$ significant digit of the parameters). The solution for 4 parameters presents the largest differences, however, one 
should notice that the PSO fitness in case (iv) is slightly smaller than the BFGS solution (i.e. PSO solution is actually slightly more accurate than BFGS by providing a better minimum).

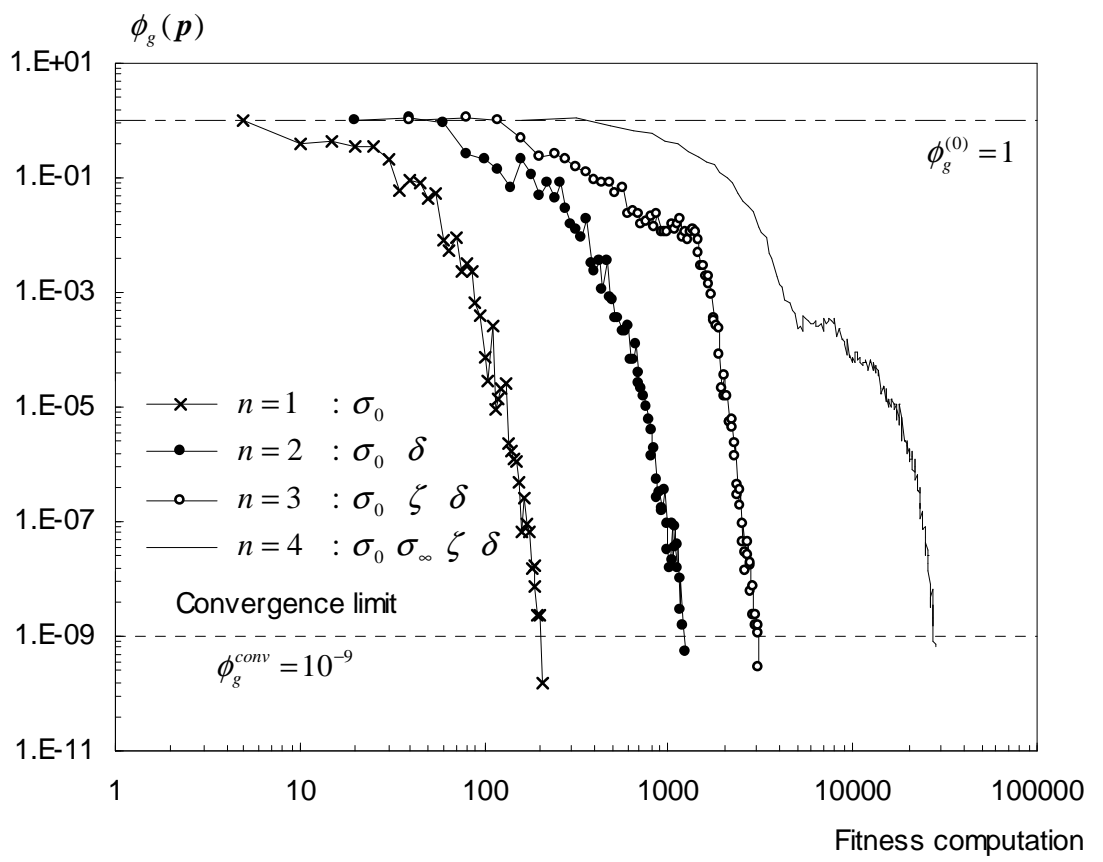

Figure 5. Computational cost: influence of the number of parameters.

Table 5. Mean equivalent number of bits, $\bar{n}_{b}^{e}$, at convergence.

\begin{tabular}{lcccccc} 
& & \multicolumn{4}{c}{ Parameter } & Mean \\
\cline { 3 - 6 } \multicolumn{1}{c}{ Case } & & $n_{\sigma_{0}}^{e}$ & $n_{\sigma_{\infty}}^{e}$ & $n_{\zeta}^{e}$ & $n_{\delta}^{e}$ & $\bar{n}_{b}^{e}$ \\
\hline (i) 1 Parameter & $n_{p}=5$ & 23.120 & - & - & - & 23.120 \\
(ii) 2 Parameters & $n_{p}=20$ & 24.374 & - & - & 23.505 & 23.939 \\
(iii) 3 Parameters & $n_{p}=40$ & 24.196 & - & 24.189 & 23.388 & 23.924 \\
(iv) 4 Parameters & $n_{p}=160$ & 23.771 & 25.170 & 24.413 & 24.016 & 24.342 \\
\hline
\end{tabular}

An alternative accuracy measure can be established by the mean equivalent number of bits, $\bar{n}_{b}^{e}$, which represents a parametric mean difference of parameters with respect to the their lower and upper limits. Theoretically, if the average difference of an individual coordinate of the hyperspace, $\Delta p_{i}$, approaches zero (i.e., the individual parameter presents the same value for all particles), the corresponding equivalent number of bits $n_{i}^{e} \rightarrow \infty$. The convergence criterion based on the normalised fitness used in this test case, $\phi_{g}^{\text {conv }}=10^{-9}$, corresponds to a mean equivalent number of bits $\bar{n}_{b}^{e} \approx 23$. Table 5 shows the equivalent number of bits for each parameter at convergence, $\left\{n_{\sigma_{0}}^{e}, n_{\sigma_{\infty}}^{e}, n_{\zeta}^{e}, n_{\delta}^{e}\right\}$, and the corresponding mean value. It is remarkable that convergence takes place with similar accuracy in those cases which $n>2$, especially when the order of magnitude is as different as $10^{8}$ and $10^{1}$ for parameters $\sigma_{\mathrm{o}}$ and $\delta$, respectively. 


\subsection{PSO / GA comparative assessment}

Genetic Algorithms are a subset of so-called evolutionary algorithms, in which each potential solution (individual) is represented by a vector of design variables (chromosome) and each design variable (gene) is described by an information encoding system (e.g. binary encoding). The classical literature [22] indicates that GA are potentially able to avoid local minima, leading to trustworthy results in non-convex problems. However, their well-known low convergence rate is a clear disadvantage. Despite widespread use in general optimization problems, application of GA to identification of inelastic parameters is relatively recent, as shows references [3-5]. More importantly, most works associate GA with other optimization strategies (e.g. gradient-based schemes) aiming at reducing the search space and providing initial estimates close to optimum. The present work adopts the GA method described in reference [8] with the following control definitions: 25 bits, $5 \%$ mutation rate, one-point crossover with a probability of $80 \%$ and full elitism. The results are summarised in Figure 6 and Table 6, which present GA / PSO fitness evolution and corresponding parameters after 400 generations. The GA / PSO population size used in this example is $n_{p}=160$.

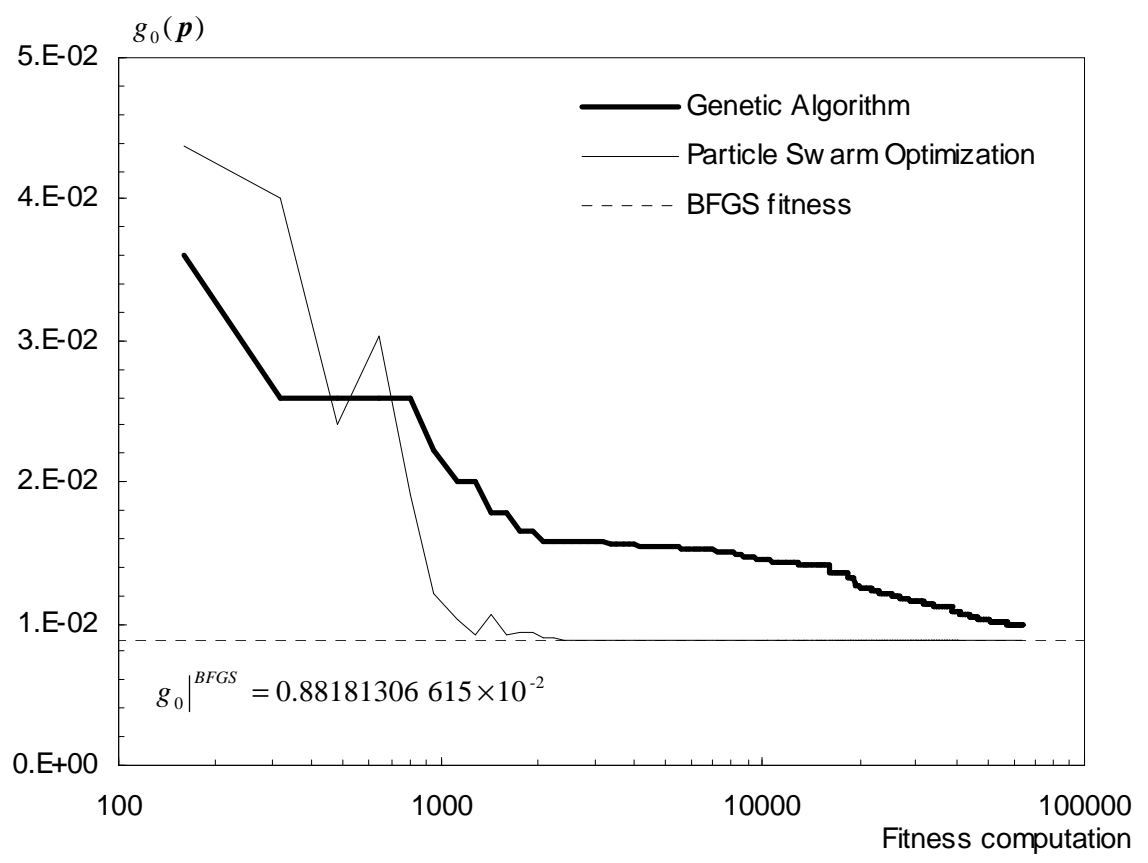

Figure 6. Fitness evolution for GA and PSO method for a population size $n_{p}=160$.

Figure 6 shows that GA provides a higher convergence rate in the first few generations. However, as the optimization progresses, convergence rate decreases substantially so that, even for 400 generations, material parameters still present relevant differences with those determined by the BFGS and PSO techniques, as indicated in Table 6. Interestingly, the mean equivalent number of bits at this stage is $\left.\bar{n}_{b}^{e}\right|^{\text {GA }} \approx 7$, significantly smaller than its PSO counterpart, $\left.\bar{n}_{b}^{e}\right|^{P S O} \approx 23$, at convergence. The authors performed several test runs for the present example, but the slow convergence has rendered the GA scheme inappropriate to be used with the objective of finding the final parameters with higher accuracy. In spite of such differences, the corresponding GA load - elongation curve shows good approximation to the experimental 
data, as exhibited in Figure 7. The PSO final curve and differences, $\varphi=R_{q}^{E x p}-R_{q}^{F E M}$, are also indicated in Figure 7.

Table 6. Parameters and fitness for GA and PSO methods at step / generation 400.

\begin{tabular}{cccccc}
\cline { 2 - 5 } Method & $\sigma_{0}[\mathrm{MPa}]$ & $\sigma_{\infty}[\mathrm{MPa}]$ & $\zeta[\mathrm{MPa}]$ & $\delta$ & $g_{0}(\boldsymbol{p})$ \\
\hline$B F G S$ & 471.2533 & 678.1928 & 218.1305 & 15.52408 & $0.88181306615 \times 10^{-2}$ \\
\hline$P S O$ & 471.2359 & 678.1867 & 218.1493 & 15.52628 & $0.88181304177 \times 10^{-2}$ \\
$G A$ & 427.7568 & 665.9593 & 261.7742 & 20.84847 & $0.98507858615 \times 10^{-2}$ \\
\hline
\end{tabular}

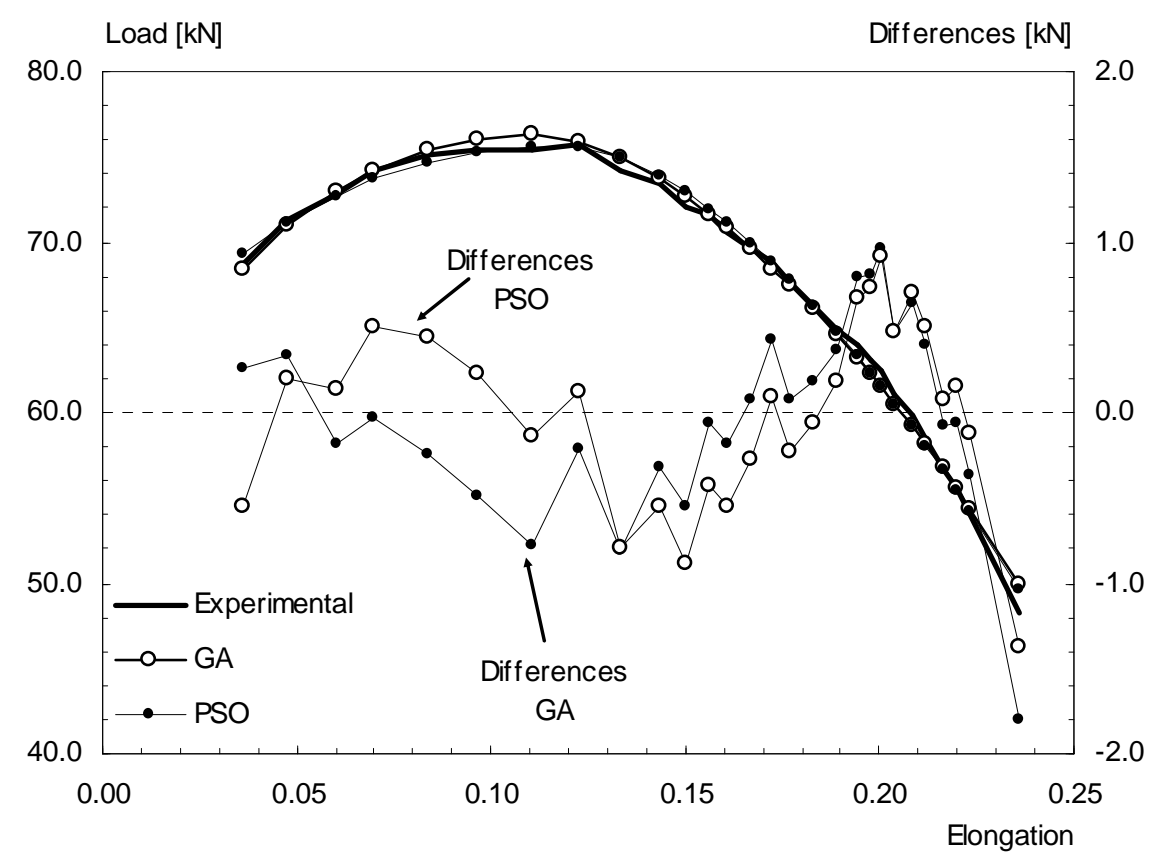

Figure 7. Load - elongation curve: final PSO and GA solutions and respective, $\varphi=R_{q}^{E x p}-R_{q}^{F E M}$, differences.

\section{CONCLUDING REMARKS}

In recent years, identification of material parameters using optimization techniques has been suggested as a viable complement to experimental measurements. The literature shows that most parameter identification strategies use either gradient-based optimization approaches (e.g. the BFGS method) or heuristic techniques (e.g. Genetic Algorithms). Despite the reported success in application of Particle Swarm Optimization to several engineering problems, very few works approach identification of material parameters using this technique. This study addresses application of the PSO scheme to this class of problems. Assessment of the PSO method uses the experimental tensile test data provided by Ponthot and Kleinermann [19], from which four hardening parameters of the modified Voce's [20] yield stress curve is determined. A reference BFGS solution for the test case is also presented. The simulations 
show that the final material parameters determined by the PSO technique are very accurate when compared against the BFGS method. The study has also determined that identification of larger number of parameter requires larger populations to avoid sub-optimal solutions. In the present case, a population of 160 particles was able to determine the optimum material parameters. A brief comparison of GA and PSO schemes indicates that, despite good approximation of the load - elongation curve, the former was not capable to converge with the same level of accuracy of the PSO method.

\section{Acknowledgements}

The first author acknowledges the research grant provided by CNPq (National Council for Scientific and Technological Development - Project 301991/2009-0). The authors also thank J. Stahlschmidt for his role in implementing the BFGS method.

\section{REFERENCES}

[1] Muñoz-Rojas, P.A., da Cunda, L.A.B., Cardoso, E.L., Vaz Jr., M. and Creus, G.J., “A mixed optimization approach for parameter identification applied to the Gurson damage model”, in Vaz Jr., M., de Souza Neto, E.A. and Muñoz-Rojas, P.A. (Eds.), Advanced Computational Materials Modeling: from Classical to Multi-scale Techniques, WileyVCH, pp.165-204, 2011.

[2] Abendroth, M., Kuna, M., "Identification of ductile damage and fracture parameters from the small punch test using neural networks", Engineering Fracture Mechanics, 73, 710-725, 2006.

[3] Aguir, H., Belhadjsalah, H., Hambli, R., "Parameter identification of an elasto-plastic behaviour using artificial neural networks-genetic algorithm method", Materials and Design, 32, 48-53, 2011.

[4] Chaparro, B.M., Thuillier, S., Menezes, L.F., Manach, P.Y., Fernandes, J.V., "Material parameters identification: gradient-based, genetic and hybrid optimization algorithms", Computational Materials Science, 44, 339-346, 2008.

[5] Muñoz-Rojas, P.A., Cardoso, E.L., Vaz Jr., M., "Parameter identification of damage models using genetic algorithms", Experimental Mechanics, 50, 627-634, 2010.

[6] Eberhart, R.C., Kennedy, J., "A new optimizer using particle swarm theory", in: Proceedings of the Sixth International Symposium on Micro Machine and Human Science, IEEE Press, 39-43, 1995.

[7] Kennedy, J., Eberhart, R.C., "Particle Swarm Optimization", in: Proceedings of the IEEE International Conference on Neural Networks, IEEE Press, 1942-1948, 1995.

[8] Vaz Jr., M., Cardoso E.L., Stahlschmidt, J., "Particle Swarm Optimization and identification of inelastic material parameters", Submitted for publication, 2011.

[9] de Souza Neto, E.A., Perić, D., Owen, D.R.J., Computational Methods for Plasticity. Theory and Applications, Wiley, 2008.

[10] Poli, R., Kennedy, J., Blackwell, T., "Particle swarm optimization - An overview", Swarm Intelligence, 1, 33-57, 2007.

[11] Blum, C., Li, X., "Swarm intelligence in optimization", in: Blum, C. and Merkle, D. (Eds.), Swarm Intelligence - Introduction and Applications, Springer, 43-85, 2008.

[12] Sedighizadeh, D., Masehian, E., "Particle swarm optimization methods, taxonomy and 
applications", International Journal of Computer Theory and Engineering, 1, 17938201, 2009.

[13] Ardakani, M.D., Khodadad, M., "Identification of thermal conductivity and the shape of an inclusion using the boundary elements method and the particle swarm optimization algorithm", Inverse Problems in Science and Engineering, 17, 855-870, 2009.

[14] Cortes, O., Urquiza, G., Hernandez, J.A., "Inverse heat transfer using LevenbergMarquardt and particle swarm optimization methods for heat source estimation", Applied Mechanics and Materials, 15, 35-40, 2009.

[15] Tian, N., Sun, J., Xu, W, Lai, C.-H., "Quantum-behaved particle swarm optimization with ring topology and its application in estimating temperature-dependent thermal conductivity”, Numerical Heat Transfer, Part B, 60, 73-95, 2011.

[16] Schutte, J.F., Groenwold, A.A., "A study of global optimization using particle swarms", Journal of Global Optimization, 31, 93-108, 2005.

[17] Clerc, M., Kennedy, J., "The particle swarm-explosion, stability, and convergence in a multidimensional complex space", IEEE Transaction on Evolutionary Computation, 6, 58-73, 2002.

[18] Rao, S.S., Engineering Optimization. Theory and Practice, fourth ed., Wiley, 2009.

[19] Ponthot, J.-P., Kleinermann, J.-P., "A cascade optimization methodology for automatic parameter identification and shape/process optimization in metal forming simulation", Computer Methods in Applied Mechanics and Engineering, 195, 5472-5508, 2006.

[20] Voce, E., "The relationship between stress and strain for homogeneous deformation", Journal of Institute of Metals, 74, 537-562, 1948.

[21] Arora, J.S., Introduction to Optimum Design, second ed., Elsevier, 2004.

[22] Goldberg, D., Sastry, K., Genetic Algorithms: The Design of Innovation, Springer, 2011. 